\title{
The Review About Method of Ultrasonic Detection of Concrete Damage Guojun $\mathrm{Liu}^{1, \mathrm{a}}$, Zeyou $\mathrm{Hu}^{1, \mathrm{~b}}$ and Dahuan Wang ${ }^{1, \mathrm{c}}$ \\ ${ }^{1}$ College of Civil Engineering, Sichuan Agricultural University, China \\ a93304276@qq.com, b123053841@qq.com, c1664792318@qq.com
}

Keywords: Ultrasonic method, detection, concrete damage, acoustic parameters

\begin{abstract}
This paper points out the basic principle of ultrasonic measuring damage, expounding the development and the research status of ultrasonic detection of concrete damage. It also summarizes the development trend of the method and makes suggestions to the further study of the technology. So it is referable for engineering technical personnel who want to know the method comprehensively and systematically.
\end{abstract}

\section{Introduction}

Concrete exists large amount of micro defects such as micro pore and interface crack before under external load due to drying shrinkage, exudation, heterogeneity of concrete material and the complex physical state, and the distribution of these defects are completely random. Under the action of outside environment and the load, the structure or the material's degradation process with the development of mesoscopic hierarchy of micro defects is referred to as damage. Damage of concrete can be summarized into five major research levels: elastic and plastic damage, static and dynamic damage, isotropic and anisotropic damage, macroscopic phenomenological and mesoscopic damage, localization and nonlocal damage.

Now nondestructive testing methods for stress damage degree of concrete include the acoustic emission method, ultrasonic pulse method, electron microscope method, photoelastic patch method and so on, and the ultrasonic pulse method is one of the most common methods. Ultrasound has a wide application in nondestructive testing of concrete, at the same time the study of ultrasonic detection of concrete damage is in-depth. Ultrasonic wave velocity and amplitude of acoustic parameters can reflect the mechanical state of concrete, simple and convenient to estimate properties and mechanical properties of concrete structure, but also can realize and measure its internal damage, which play an important role in the diagnosis of concrete structures, repair and maintenance.

\section{The Characteristics of Ultrasonic Testing in the Concrete and the Basic Principle of Ultrasonic Measuring Damage}

Because the concrete is elastic and plastic material, which composed of solid, liquid and gas. its internal interface distribution is extremely complicated, making the ultrasonic transmission also has unique properties that determine the ultrasonic testing of concrete with the following characteristics: (1) Multiphase exists in the concrete and the difference of acoustic resistance among each other is significant, causing scattering attenuation. Scattering attenuation have a greater impact on high frequency ultrasonic, so using low frequency ultrasonic is reasonable; (2) Low frequency transducer launch beam approximation the spherical wave, at the same time stones and other substances exist irregular interface. These reasons cause the phenomenons such as refraction, reflection and diffuse reflection when ultrasonic propagates in concrete, which make the sound directivity poorer; (3) Because of the refraction and reflection, the transmission of ultrasonic wave in the concrete internal is not a straight line; (4) The ultrasonic longitudinal wave propagates in the concrete. The longitudinal wave and transverse wave producing along the way will overlap and interfere each other in different phase, different path, causing the received ultrasonic signal very complex.

Ultrasonic instrument transmitter converter launchs ultrasonic pulse discontinuity by repetitive frequency, which is the ultrasound. In the concrete of intact, ultrasonic wave velocity is relevant to 
concrete elastic modulus, density, poisson's ratio and so on. After the damage, the generation of cracks make the phenomena such as the transmission of sound wave diffraction, reflection, reducing the acoustic propagation path or making energy lower. These information can reflect through acoustic parameters, so they can be used to condition assessment of concrete damage.

According to the detection principle, ultrasonic flaw detection is divided into penetration method and reflection method. At present, penetration method mainly adopted. Penetration method is to use an emission transducer emiting ultrasonic pulse repeatedly, making ultrasonic wave propagate in the concrete which is under detection. And it is received by receiving transduceris after the ultrasonic wave received is converted into electrical signals which will be amplified through ultrasonic instrument amplifier. Finally, measuring the received ultrasound signal, and the acoustic parameters are displayed on the oscilloscope screen. The use of the relative change of pulse wave propagation speed, receiving the amplitude and frequency of the acoustic parameters in the technical conditions of the same concrete and relative comparison to judge the damage of concrete and its defects is the basic basis of using of ultrasonic flaw detection of concrete damage and internal defects.

\section{The Research Status of Ultrasonic Detection of Concrete Damage}

Since some researchers who come from Canada, Germany and the UK used ultrasonic pulse detection technology for the first time in the concrete structure detection in 1949, the nondestructive testing technique has been widely applied. However, due to many of the uncertainty factors in the strength testing, it had led to the technology of ultrasonic measuring intensity developing slowly. To the end of the $1970 \mathrm{~s}$, with the development of electronic technology, ultrasonic detector continuously improved and perfected which promoted the development of ultrasonic testing technology. Today,with the ultrasonic detecting instrument constantly updated and the heigh development of computer technology,ultrasonic pulse testing technology has become one of the important means of engineering structure damage detection.

Damage Detection of Concrete Members under Cyclic Loading. Circular damage is caused by repeated stress effect, and is the function of cycles. At present most scholars to research and analysis of concrete damage, most are under the effect of static damage characteristics of materials or structures. But in recent years as more destructive earthquake occurred, the research of the law of structural dynamic damage development is deepening. Earthquake as random dynamic load acting on the structure make the strength of the structure and mechanical properties change complexity. At present there are cumulative damage methods of earthquake damage assessment model, such as based on the deformation and cumulative damage model, based on energy accumulation model and hybrid model. How to reflect the damage of the earthquake damage about these models by field testing and calculation is a very important problem. Liao Jiehong et al defined the degree of damage coefficient by displacement and stiffness information and defined damage coefficient by the acoustic wave velocity and wave amplitude information. Flat of concrete column and short limb shear wall, a total of three concrete members pseudo-static test, were measured about ultrasonic acoustic parameters through the whole process that they obtained wave velocity and amplitude changed with the loading force and displacement under the condition. Finally, according to this test they got the relationship between the acoustic parameters and damage coefficient. The test showed that using ultrasonic acoustic parameters change can detect earthquake damage of concrete component. But due to the number of specimens in only three, geting the component itself damage coefficient and acoustic damage coefficient under various conditions of relational expression of coefficient remains to be further improved.

The Detection of Crack Depth of Concrete. The concrete is often the firstly destroyed from micro defects existing in the concrete internal destructive under load, gradually developing into the other parts. Finally, it forms the macroscopic crack and fracture damage, so it is particularly important to formulate the corresponding remedial measures by measuring the crack width and depth. Ultrasonic single side method of measurement is relatively common, simple, rapid and suitable for the engineering practice, so the detection of crack depth often is used by the ultrasonic level measurement method. There is the lack of systematic and large amounts of lab and field test data in 
many of the existing research results, and it is not enough understanding for the influence and the accuracy of tests. In view of above factors, Tian Hui et al made research for the reliability of ultrasonic method of measuring the depth of fissures of detection through the indoor model test, and made it clear that reliability and accuracy of the concrete component standard cracks through the ultrasonic detection were reasonable. In consideration of the influent factors about the crack containing water and impurities, the line of measuring and the crack oblique crossing and analyzing the crack depth through asymmetric arrangement measuring points position of transducer and so on, pointing out that the above factors have certain influence on test results, but the relative error satisfies the requirement of engineering testing. Guo Weiling et al have revised in ultrasonic actual path. For the depth of vertical cracks between 50 250 mm through the experiment, when it is not reinforced influence, the crack depth measurement precision is relatively high whose error within $5 \%$ by using ultrasonic level measurement method. However, in pact the influence of reinforcement on the depth of crack can not be ignored, Hao Shihua made a field test for the cement concrete slab crack, and the test data has been got by using the numerical average method and a way of eliminating method. The test results have been combined with a concrete crack discriminant standard which has carried on the comprehensive judgement of crack depth. they pointed out that when there were effects of reinforced concrete structure layer distribution, two transducer connection with steel were best into Angle of $40^{\circ}$ to $45^{\circ}$ and the test effect would be better.

Damage Detection of Concrete Action by the Impact. The working mechanism of concrete material impact damage is complex. The quantitative basis for the safety assessment of after injury is insufficient, and the lack of quantitative and rational analysis of the project would result in the assessment of risks. Therefore, finding a way evaluates effectively the degree of concrete damage after impact, which having great significance to evaluate the residual bearing capacity and to determine the repair plan. Researching on the dynamic impact of concrete at home and abroad has made many results, but some studies did not consider the effect of loading history on concrete mechanics performance, and the others did not reflect the impact instant loading effect. Tian Yubin et al $^{[1]}$ used ultrasonic detection to the concrete prism specimens before and after impact and proposed damage variable $\mathrm{D}$ based on ultrasonic velocity changes, which can detect the extent of damage when their appearance have no significant changes. Besides, the stress-strain curve of damaged concrete materials could be got by axial compression test, and an estimate method for calculating residual strength of damaged concrete materials was also obtained.

The Testing of Damage Depth of Concrete After Firing. In recent years, fire accident have been increasing in China. The occurrence of fire not only caused great losses of life and property, but also led to the different degree damage of building structures. The detection or diagnosis for crack depth is the important basis of comprehensive evaluation when the concrete structure was damaged after firing. Propagation velocity of ultrasound in concrete is not only related to the porosity, but also related to the moisture content of concrete. The moisture evaporate inside the concrete when the concrete structure is by the fire, then the porosity and density change that affecting the ultrasonic velocity ultimately. Therefore, you can characterize the damage degree of concrete according to the changes of sound velocity.

Technical specification for concrete defects of ultrasonic testing (CECS 21: 2000) assumes that concrete damage is a two stitches model and the path of acoustic wave propagation is a tri-model. This assumption is quite different with the actual situation, because in practice it is difficult to find a more accurate two stitches intersection, causing difficulties in the analysis. Liao Jiehong et al did research based on the assumption of concrete damaged trends hyperbolic model in the literature [2], with the first wave propagation path parabolic improved the algorithm. With ABAQUS finite element software to simulate the acoustic wave propagation in the damaged concrete, the improved algorithm was validated. The results showed that the improved algorithm had higher accuracy and was more realistic. Chen Tianhong et al studied the universality of different types of concrete after high-temperature processing with ultrasonic evaluation, and the research told that different types of concrete showed the an identical result after high-temperature processing evaluated by using damage and performance of the relative velocity. Many existing outcomes lack a complete study on the 
durability damage of concrete under high temperature. Wang Hailong et al studied the use of ultrasonic nondestructive testing methods to evaluate concrete damage after high temperature in the literature [3] and established innovatively correspondence between ultrasonic indicators of change and concrete durability after disaster, as well as provided a reliable basis for the non-destructive testing and fast assessment in the fire scene of concrete.

\section{The Development Trend of Ultrasonic Detecting Method of Concrete Damage}

We can get certain conclusions by ultrasonic testing way to concrete damage location and the judgment, however, we can only roughly reflect the test part of the average condition, but not to give deterministic quantitative and the description to the damage of attribute parameters such as the scope, size and shape. Ultrasonic CT is a kind of computer tomography technology which is a rapid development of modern nondestructive testing technology, whose theoretical basis is from medical CT imaging technology. This emerging technology via specific digital image processing technology can display the concrete internal state comprehensively and completely, which are more convenient for defect detection and damage detection. And Liu Wen pointed out in the literature that ultrasonic CT testing is more suitable for large concrete structures, which is a gratifying results. Because in real life, most of the concrete structure is large, it will be conducive to the application of ultrasonic CT testing in practical engineering.

\section{Conclusions and Recommendations}

Ultrasonic detection method with other detection methods, such as acoustic emission method, should complement each other, making test results more accurate and reasonable.

Many existing achievements also lack a complete research on the laws of the durability of concrete under high temperature damage. We should vigorously carry out research of durability damage of concrete under high temperature.

Concrete damage analysis of the current form of study, most of the scholars study under the action of static damage characteristics of structure, so the law of development of structure's dynamic damage should deeply research as well.

This paper introduces the method of ultrasonic detection of concrete damage development situation, basic principle and research status, pointing out the method of ultrasonic detection of concrete damage and making recommendations for trend of future research directions. This article for the technology and technical personnel getting a system and comprehensive understanding and who want to further study the technology has a certain reference value.

\section{Acknowledgments}

This work was supported by a grant from the National Natural Science Foundation of China, whose name is "Research on Fatigue Damage Model for Concrete Based on Residual Strain and Failure load model of vehicles" (No. 51408385).

\section{References}

[1] Tian Yubin, Huang Tao, Liu Jia, Jin Ruoxi, Zhang Chunwei. Damage detection and compressive behavior of concrete after impacting[J]. Journal of Building Structures, 2014, S1:58-64.

[2] Liao Jiehong, Yu Jiangtao, Lu Zhoudao. Ultrasonic Testing Algorithm to the Surface Damage of Fire-Damaged Concrete[J]. Non-destructive Testing, 2011, 03:19-22 + 39.

[3] Wang Hailong, Yu Qiujia, Sun Xiaoyan, Chen Kexing. Durability and damage evaluation of concrete subjected to high temperature. Jiangsu University (Natural Science), 2014, 02:238-242. 